\title{
Complications associated with postoperative dressings: a clinician's perspective
}

\author{
Upton P, Dunk AM \& Upton D
}

Keywords Adhesives, dressings, skin injury

For referencing Upton $\mathrm{P}$ et al. Complications associated with postoperative dressings: a clinician's perspective. Wound Practice and Research 2019; 27(4):158-163.

DOI https://doi.org/10.33235/wpr.27.4.158-163

\section{ABSTRACT}

Background Medical adhesive-related skin injuries (MARSI) are a relatively new category of skin trauma recognised to occur in postoperative settings. Protecting against MARSI is important since they may impact negatively on patient outcomes and significantly add to the cost of treatment. Despite this, evidence to guide best practice in postoperative wound care dressings is limited. In this study we therefore aim to quantify clinicians' perceptions of the frequency and severity of postoperative MARSI.

Method Australian clinicians with experience of working with postoperative wounds in the past 12 months completed an anonymous online questionnaire.

Results Over a quarter (27\%) of patients with postoperative wounds were estimated to have experienced a MARSI over the past 12 months. Complications were least likely to be associated with silicone adhesives and were most frequently

Penney Upton*

MA, MPhil, PGCHE, PhD

Associate Professor, Centre for Research and

Action in Public Health (CeRAPH),

University of Canberra, ACT

Email Penney.upton@canberra.edu.au

Ann Marie Dunk

RN Cert Wound Mt, BHlthSc (Nurs), MN (Research) Clinical Nurse Consultant, Tissue Viability Unit, Canberra Hospital, ACT

\section{Dominic Upton}

BSc, MSc, PhD

Dean, College of Health \& Human Sciences,

Charles Darwin University, NT

* Corresponding author associated with polyurethanes and acrylates. The most frequent complication observed was pain.

Conclusion Whilst it is reassuring that complications are less likely to be associated with silicone, a commonly used dressing adhesive, it is of concern that two other frequently used adhesives - acrylates and polyurethanes - have the highest association of complications. Shifting to using more silicone adhesives could reduce the economic burden of postoperative complications and improve patient outcomes by reducing MARSI-associated pain.

What is already known on this topic

Complications from the effective management of surgical wounds may arise from the dressing itself, particularly the adhesive used to keep the dressing on the skin, although the exact nature and extent of these is still to be investigated. Whilst our understanding of MARSI is emerging, the evidence to preference the use of one wound dressing over another is limited. Given the potential human and economic costs of postoperative complications, it is important that the nature of surgical site healing complications related to different dressing types continues to be investigated.

\section{What this manuscript contributes}

This paper reports the observations of Australian clinicians - predominantly nurses - involved in postoperative wound care. Importantly, our findings provide estimates of:

- The extent of MARSI for postoperative wounds in Australia.

- The most common types of MARSI seen in postoperative wound care.

- The type of complication associated with different adhesives.

\section{BACKGROUND}

The World Health Organization (WHO) describes surgical care as "an indispensable component of a functioning health system"1. However, surgery is associated with a 
high economic cost related to the need for skilled human resources, specialist supplies and infrastructure so, perhaps not surprisingly, the majority of operations $(59 \%)$ take place in high-income countries ${ }^{1}$. However, this essential component of health management has been observed to increase on an annual basis across both high- and low-income countries; from 2004-2012 this increase was calculated to be in the region of $38 \%$, with the total global volume of operations estimated to be between 266.2-359.5 million'. In Australia, $25 \%$ of hospitalisations involve some form of surgery, with 2.7 million - both elective and emergency - operations having taken place in 2015-2016².

Postoperative wound care plays an important role in reducing the complications associated with surgical wound healing, including surgical site infection, wound dehiscence and/ or haematomas ${ }^{3}$. Protecting against healing complications after surgery is important since these may increase length of hospital stay, negatively impact on patient outcomes, and significantly add to the cost of treatment ${ }^{4,5}$.

Surgical wounds heal by primary intention when the wound edges are brought together and secured ${ }^{6}$. Often wound dressings are also applied after a wound is closed. Use of dressings in this way generally assumes that they provide a barrier from environmental contamination, reducing the risk of infection ${ }^{4}$. Wound dressings may also absorb exudate, offer physical support and protection, facilitate wound observation, and meet a patient's desire that their wound be covered ${ }^{4,6}$.

However, whilst wound dressings therefore provide some benefit for postoperative patient care, they may also add to postoperative complications. It has long been recognised that skin trauma may occur as a result of dressing adhesives, although the categorisation of these as medical adhesiverelated skin injuries (MARSI) is relatively new ${ }^{7}$. MARSI are recognised to occur across all care settings, and to play a significant role in patient safety ${ }^{8}$. MARSI occur when superficial layers of skin are removed by the medical dressing adhesive, resulting in threats to skin integrity, which include skin tears, or a reaction such as the formation of vesicles. They also cause pain, increase the risk of infection, may increase wound size, and delay healing.

There are many different types of wound dressings available ${ }^{9}$. However, to date, there is limited evidence to preference the use of any one specific wound dressing type postoperatively, particularly in regard to the avoidance of MARSI ${ }^{4,6}$. Indeed, there is generally a lack of evidence to guide best practice in post-surgical wound care dressings ${ }^{10,11}$. Studies that have been undertaken generally include small sample sizes, low event rates, and are at high or unclear risk of bias ${ }^{4,6}$. This lack of clear evidence led the most recent Cochrane review to recommend that decisions on postoperative wound dressings be based on dressing costs and the need for management of specific symptoms such as absorption of exudate $^{6}$.
Postoperative care may also include the use of a peripheral intravenous (IV) device. Complications from IV sites have also been highlighted as a risk to patient care, particularly in relation to infection ${ }^{12}$. This is thought to result either from normal skin flora bacteria migrating into the puncture wound at the time of insertion, or from the penetration of outside organisms post-insertion. Thus, there are two key components to IV site management if complications are to be avoided - the skin disinfection method used before the IV is inserted, and the type of dressing used to cover the site and protect it ${ }^{12}$.

Given the limited evidence available regarding postoperative wound/IV dressings, it is important that the nature of surgical site healing complications and use of different dressing types continues to be investigated. The experience of clinicians who work with postoperative wounds is an important aspect of evidence which should be considered. For example, it is essential to know if actual practice is in line with that which is agreed ideal in policy or practice terms and, if not, why not. This study was therefore designed to quantify clinicians' perceptions of the frequency and severity of postoperative wound healing complications - including IV wounds - and associations with the use of different dressing adhesives. Specifically, it aimed to address the following research questions:

- What is the frequency and severity of post-surgical primary intention wound healing complications that can be linked, at least in part, to dressing adhesives?

- What is the frequency of post-surgical primary intention wound healing complications associated with each dressing adhesive type?

- What is the frequency and severity of IV wound complications that is linked, at least in part, to dressing adhesives?

- What is the frequency of IV wound complications associated with each dressing adhesive type?

We also sought to describe:

- How decisions are made about the use of specific dressing types within participants' workplaces.

- How frequently each dressing and adhesive type is used, both in the management of post-surgical wounds healing by primary intention and the management of IV sites.

- If participants had received training and education in the use of different dressing types and, if so, where from.

\section{METHOD}

Participants were recruited using self-selection sampling methods via an embedded survey link on the Wounds Australia website. Wounds Australia is the peak body for wound care and management in Australia, and engages in advocacy, education and research. Potential participants were also contacted via the Wounds Australia members' emailing list. Eligible participants were clinicians who had had experience of working with postoperative wounds and/ 
Table 1. Mean estimated proportion of patients experiencing specific complications linked to dressing adhesives.

\begin{tabular}{|l|l|}
\hline Complication & Percentage of patients \\
\hline Pain & $30 \%$ \\
\hline Irritant contact dermatitis & $27 \%$ \\
\hline Maceration & $21 \%$ \\
\hline Skin stripping & $21 \%$ \\
\hline Tension injury/blister & $20 \%$ \\
\hline Allergic dermatitis & $17 \%$ \\
\hline Skin tear & $14 \%$ \\
\hline Infection & $13 \%$ \\
\hline Dehiscence & $9 \%$ \\
\hline Folliculitis & $7 \%$ \\
\hline
\end{tabular}

or IV wounds in the past 12 months. Ethical approval for the study was provided by the University of Canberra Human Research Ethics Committee (project number 17-166).

\section{RESULTS}

Surveys were completed by 100 respondents, of which 77 were eligible for analysis (mean age 45 years). The sample comprised predominantly female (93\%) nurses (95\%). Participants were recruited from across Australia, with surveys submitted predominantly from Queensland $(31 \%)$, the Australian Capital Territory (24\%) and Victoria (22\%).

Perceived frequency and severity of post-surgical primary intention wound healing complications linked to dressing adhesive

According to survey respondents, an estimated $27 \%$ of all patients with postoperative wounds whom they had cared for over the past 12 months had experienced complications linked, at least in part, to the dressing adhesive, with $8 \%$ of these infections being rated as severe. In addition, $27 \%$ of patients with fragile skin were estimated to have experienced healing complications caused, at least in part, by the dressing adhesive. As demonstrated in Table 1, pain was believed to be the most frequently experienced complication, closely followed by irritant contact dermatitis.

Perceived frequency of post-surgical primary intention wound healing complications associated with each dressing type

As demonstrated in Table 2, complications were perceived to be most frequently associated with rubber-based adhesives, polyurethanes and acrylates. They were least likely to be associated with silicone or hydrogels.

The type of complication most commonly associated with each adhesive is shown in Table 3. This shows that irritant contact dermatitis, skin stripping, tension injuries, skin
Table 2. Mean estimated proportion of complications linked to specific dressing adhesives.

\begin{tabular}{|l|l|} 
Adhesive type & $\begin{array}{l}\text { Percentage of } \\
\text { complications }\end{array}$ \\
\hline Rubber-based adhesive & $35 \%$ \\
\hline Polyurethane & $28 \%$ \\
\hline Acrylates & $22 \%$ \\
\hline Hydrocolloids & $12 \%$ \\
\hline Silicone & $10 \%$ \\
\hline Hydrogels & $6 \%$ \\
\hline
\end{tabular}

tears were perceived to be more commonly associated with acrylate and polyurethane than any other adhesive. Maceration was more commonly linked to hydrocolloids and acrylates.

Perceived frequency and severity of IV wound complications linked to dressing adhesive

According to survey respondents, an estimated $25 \%$ of all patients with IV wounds whom they had cared for over the past 12 months had experienced complications linked, at least in part, to the dressing adhesive, with $9 \%$ of these being rated as severe. As demonstrated in Table 4, the common complications were irritant contact dermatitis, skin tears and skin stripping.

Perceived frequency of IV wound complications associated with each dressing adhesive type

Key findings for IV dressings were that the most common adhesives used for IV wounds were acrylates (38\%); therefore, unsurprisingly, complications were observed to be most commonly associated with this dressing type (20\%).

\section{Use of specific dressing types within the workplace}

Key findings regarding dressing decisions were that postoperative dressing choices were made mainly by the operating surgeon (28\%), the attending nurse $(24 \%)$, or a specialist nurse (13\%). In contrast, decision-making regarding IV device dressings was more likely to be made by the attending nurse (18\%) than the attending medical officer $(3 \%)$.

Frequency each dressing and adhesive type is used in the management of post-surgical wounds healing by primary intention

Table 5 shows that the most common types of dressing used for post-surgical wounds were waterproof dressings $(66 \%)$, island dressings $(44 \%)$ or foam dressings $(31 \%)$, whilst the most commonly used adhesives were silicone $(24 \%)$, polyurethane (20\%) and acrylates (19\%) as shown in Table 6. Rubber-based adhesives were reported to be rarely used, comprising only approximately $2 \%$ of dressing adhesives. Figure 1 shows the proportion of patients experiencing 
Table 3. Proportion of patients experiencing complications linked to specific dressing adhesives.

\begin{tabular}{|l|l|l|l|l|l|l|}
\hline Complication / adhesive type & Acrylates & Polyurethane & Hydrocolloids & Silicone & Hydrogel & Rubber-based \\
\hline Irritant contact dermatitis & $14 \%$ & $12 \%$ & $3 \%$ & $7 \%$ & $3 \%$ & $3 \%$ \\
\hline Skin stripping & $14 \%$ & $11 \%$ & $7 \%$ & $2 \%$ & - & - \\
\hline Tension injuries/blisters & $12 \%$ & $11 \%$ & $3 \%$ & - & $2 \%$ & $5 \%$ \\
\hline Skin tears & $12 \%$ & $9 \%$ & $3 \%$ & $2 \%$ & - & $3 \%$ \\
\hline Maceration & $10 \%$ & $4 \%$ & $12 \%$ & $9 \%$ & $7 \%$ & $5 \%$ \\
\hline Pain & $9 \%$ & $9 \%$ & $5 \%$ & $3 \%$ & $3 \%$ & $3 \%$ \\
\hline Allergic dermatitis & $7 \%$ & $7 \%$ & $3 \%$ & $7 \%$ & - & $3 \%$ \\
\hline Infection & $2 \%$ & - & $2 \%$ & $3 \%$ & $2 \%$ & - \\
\hline Dehiscence & $2 \%$ & - & $3 \%$ & - & - & - \\
\hline Folliculitis & - & $2 \%$ & - & - & - & $2 \%$ \\
\hline
\end{tabular}

complications for the three most commonly used dressing adhesives. This demonstrates that more complications are associated with polyurethane and acrylates than with silicone.

Frequency each dressing and adhesive type is used in the management of IV sites

Key findings for IV dressings and adhesives were that the most common dressing type used to protect IV wounds was a film dressing, with acrylates the most common adhesive (38\%).

Training and education in the use of different dressing types Less than half of all clinicians had received training in the use of different dressing types in the past 12 months. Training was predominantly provided by a dressing company representative $(38 \%)$, an in-house trainer $(24 \%)$, or another source such as an online module or a colleague $(31 \%)$. Training source was not specified by $7 \%$ of respondents.

Table 4. Mean estimated proportion of patients experiencing specific complications linked to IV dressing adhesives.

\begin{tabular}{|l|l|}
\hline Complication & Percentage of patients \\
\hline Irritant contact dermatitis & $16 \%$ \\
\hline Skin tears & $16 \%$ \\
\hline Skin stripping & $15 \%$ \\
\hline Allergic dermatitis & $13 \%$ \\
\hline Folliculitis & $10 \%$ \\
\hline Tension injury/blister & $10 \%$ \\
\hline Maceration & $7 \%$ \\
\hline Infection & $6 \%$ \\
\hline
\end{tabular}

\section{DISCUSSION}

According to the clinicians who responded to this survey, over a quarter (27\%) of patients with postoperative wounds healed by primary intent are estimated to have experienced complications linked, at least in part, to the dressing adhesive. The rate of complications was estimated to be the same for patients with fragile skin (27\%). Complications were observed to be most frequently associated with two of the most commonly used adhesives, polyurethanes and acrylates, and one rarely used adhesive, rubber. They were least likely to be associated with another commonly used adhesive, silicone.

These findings are in line with empirical studies including double-blind randomised control trials - that have demonstrated fewer complications such as allergic reactions, skin tears and pain to be associated with silicone

Table 5. Dressing type used in the management of post-surgical wounds healing by primary intention.

\begin{tabular}{|l|l|}
\hline Dressing type & Percentage used \\
\hline Waterproof dressings & $66 \%$ \\
\hline Island dressing & $44 \%$ \\
\hline Foam & $31 \%$ \\
\hline Topical negative pressure & $21 \%$ \\
\hline Film & $20 \%$ \\
\hline Hydrocolloid & $19 \%$ \\
\hline Gauze & $17 \%$ \\
\hline Calcium alginate & $17 \%$ \\
\hline Transparent polyamide & $15 \%$ \\
\hline Tulle gras & $13 \%$ \\
\hline
\end{tabular}


Table 6. Adhesives used in the management of post-surgical wounds healing by primary intention.

\begin{tabular}{|l|l|}
\hline Adhesive & Percentage used \\
\hline Silicone & $24 \%$ \\
\hline Polyurethane & $20 \%$ \\
\hline Acrylates & $19 \%$ \\
\hline Hydrocolloid & $18 \%$ \\
\hline Hydrogels & $10 \%$ \\
\hline Rubber-based & $2 \%$ \\
\hline
\end{tabular}

dressings ${ }^{13,14}$. However, whilst it is reassuring to see that fewer complications were linked to silicone adhesives, the perception that silicone adhesive causes irritant contact and allergic dermatitis in $7 \%$ of patients and maceration in $9 \%$ is surprising. Further investigation into the extent to which this clinician perception reflects reality is warranted.

The most commonly experienced complication linked to dressing adhesive was pain, with nearly a third of patients $(30 \%)$ estimated to have experienced this complication. The fact that this estimate is higher than the overall estimate of complications $(27 \%)$ suggests that clinicians may initially have underestimated the rate of complications; it is possible that pain is regarded as an inevitable part of the postoperative process, not a complication and thus was not considered by clinicians until prompted. However, it should be noted that pain was more likely to be associated with two of the commonly used adhesives - polyurethane and acrylate than any other adhesive, and at similar rates for each $(9 \%$ of patients).
Around a quarter of patients with postoperative wounds were estimated to have experienced complications such as irritant contact dermatitis, skin stripping or maceration. The first two complications were associated particularly with the commonly used adhesives acrylates and polyurethane, whilst maceration was more frequently associated with acrylates and hydrocolloids. It is of note that rubber-based adhesive is now rarely used, due to its status as known allergen ${ }^{15}$. In contrast, the recognition of allergies associated with acrylates and polyurethane seems less common. However, feedback from surveyed clinicians suggests this may be changing. As one respondent noted, they are "seeing increasing numbers of allergic reactions" to dressing adhesives.

What is not clear from the current survey is if patients experience a combination of complications, although from the estimates provided by clinicians it seems highly likely. This warrants further investigation, with specific attention needing to be given to which complications are mostly frequently seen together, particularly in relation to the commonly used adhesives acrylate and polyurethane.

The proportion of patients observed to experience IV wound complications was similar to that of postoperative wounds (one quarter) and, as with these, complications were mostly commonly associated with acrylates, the most frequently used IV dressing adhesive. The types of complication were also similar, consisting of irritant contact dermatitis, skin stripping and skin tears. Unlike postoperative wounds, however, pain was not seen as a complication for IV device wounds. However, in regard to IV dressings, one respondent suggested that it is not the dressing adhesive which causes complications but rather "user error". For example, "the cleaning solution is not allowed to dry prior to the application

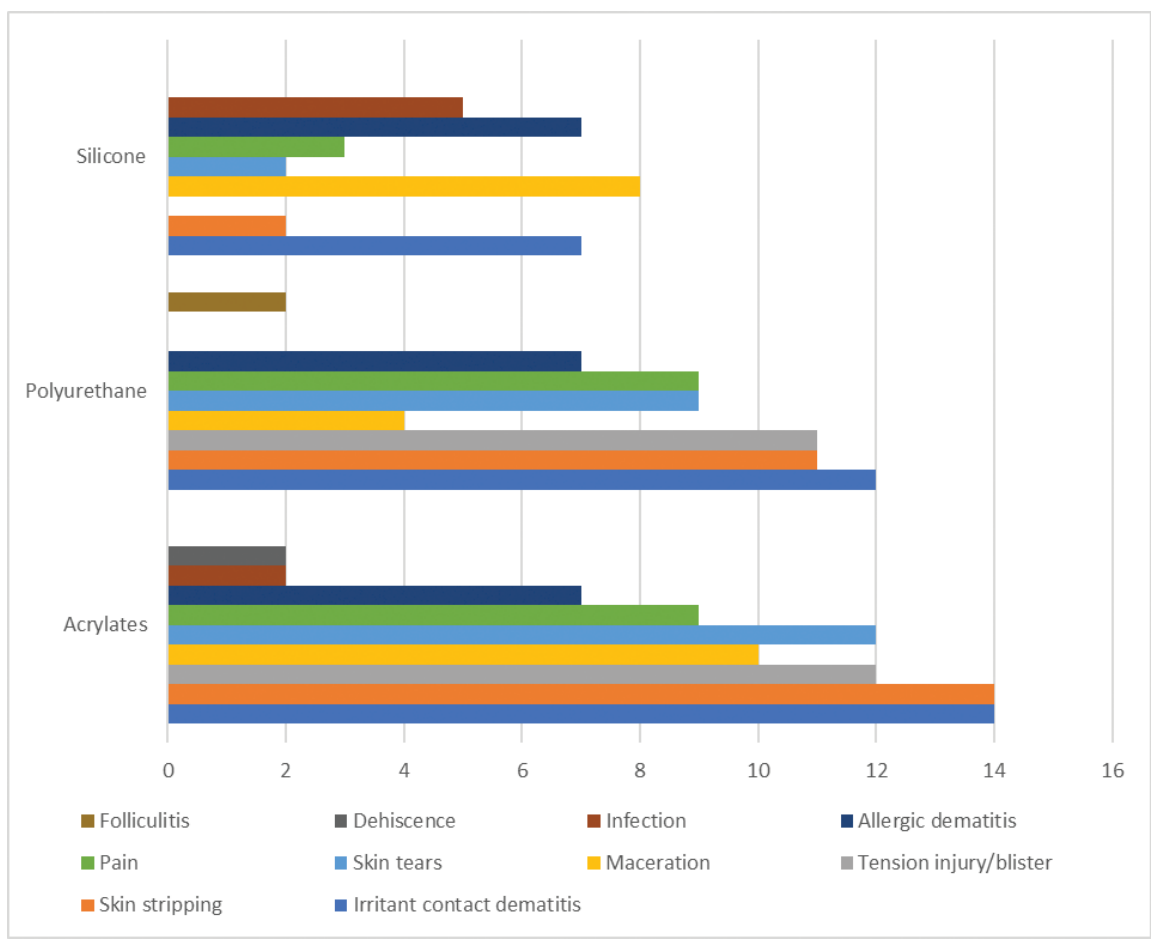

Figure 1. Proportion of patients experiencing complications linked to the most commonly used dressing adhesives. 
of the film. Or, on removal, not enough care is taken with fragile skin to prevent skin tears". This is especially the case with "inexperienced staff removing these dressings". This highlights the importance of training and education in the use of dressings; however, less than half of our sample had had any training in the application or management of wound dressings in the past 12 months.

Discussion of the impact of other possible post-surgical complications such as seromas or haematomas is beyond the scope of this paper. Although respondents were asked if they had observed seromas or haematomas resulting from dressing adhesives, these were rarely described so, for the sake of succinctness, they are not reported here. It is plausible that adhesive-related seromas and haematomas do occur but, because these are common complications following surgical intervention, it is difficult to differentiate those associated with the surgery from any resulting from the dressing adhesive. This may warrant further investigation. Finally, it should also be noted that any commentary on which dressings post-surgery may or may not aid in managing skin injuries is also out of the scope of this study as our focus was on complications arising from medical adhesive rather than their management.

\section{CONCLUSION}

Surgical intervention is a necessary, yet costly component of healthcare in the 21st century. However, as shown by this study, some of the costs associated with each episode of surgical care are avoidable since they are related to postoperative complications linked, at least in part, to dressing adhesives. According to our survey findings, over one quarter of postoperative wounds ( $27 \%$ ) had complications caused, at least in part, by a dressing adhesive, suggesting that this is a significant issue for postoperative wound care. Furthermore, if the recall of the survey respondents is accurate, then this would equate to a potential 729,000 adhesive-related complications in Australia.

Whilst it is reassuring to see that the least complications are associated with one of the more commonly used adhesives, silicone, it is of concern that two other frequently used adhesives, acrylates and polyurethanes, have the highest association of complications than any other adhesive. It is also worthy of note that the majority of IV dressings use acrylates and show a similar level of complication to other postoperative wounds. Shifting to using more silicone adhesives could therefore potentially reduce the economic burden of postoperative complications and also improve patient outcomes by reducing pain and suffering.

Finally, we identified an increased role for staff training and education in dressing use, given the suggestion that some of these problems could also be overcome with better healthcare provider knowledge and practice. Some of these could be achieved through relevant consensus statements ${ }^{15}$ and best practice guidelines such as those of the International Skin Tear Advisory panel (ISTAP) ${ }^{16}$ and Wounds UK ${ }^{17}$.

\section{ACKNOWLEDGEMENTS}

The authors would like to thank Lucy Ellen for her help in developing, distributing and analysing the survey, and Matthew Cartwright for his help in preparing this manuscript. We would also like to acknowledge Mölnlycke Health Care, without whose sponsorship this study would not have been possible.

\section{CONFLICT OF INTEREST}

The authors declare no conflicts of interest.

\section{FUNDING}

The authors received no funding for this study.

\section{REFERENCES}

1. Weiser TG, Haynes AB, Molina G, Lipsitz SR, Esquivel MM, Uribe-Leitz T, et al. Size and distribution of the global volume of surgery in 2012. Bull World Health Org 2016;94(3):201.

2. Australian Institute of Health and Welfare. Australia's hospitals 20152016 at a glance [Internet]. Canberra: AlHW; 2017 [cited 2019 Jun]. Health Services Series no. 77. Available from https://www.aihw.gov. au/getmedia/d4e53b39-4718-4c81-ba90-b412236961c5/21032.pdf. aspx?inline=true

3. Yao K, Bae L, Yew WP. Post-operative wound management. Aust Family Physic 2013 Dec;42(12):867.

4. Walter CJ, Dumville JC, Sharp CA, Page T. Systematic review and meta-analysis of wound dressings in the prevention of surgical-site infections in surgical wounds healing by primary intention. Br J Surg 2012 Sep;99(9):1185-94.

5. Badia JM, Casey AL, Petrosillo N, Hudson PM, Mitchell SA, Crosby C. Impact of surgical site infection on healthcare costs and patient outcomes: a systematic review in six European countries. J Hosp Infect 2017 May 1;96(1):1-5.

6. Dumville JC, Gray TA, Walter CJ, Sharp CA, Page T. Dressings for the prevention of surgical site infection. Cochrane Database Syst Rev 2014(9).

7. Yates S, McNichol L, Heinecke SB, Gray M. Embracing the concept, defining the practice, and changing the outcome. J Wound, Ostomy Continence Nurs 2017;44(1):13-17.

8. Ratliff CR. Descriptive study of the frequency of medical adhesiverelated skin injuries in a vascular clinic. J Vascular Nurs 2017;35(2):8689.

9. Weller C, Sussman G. Wound dressings update. J Pharmacy Pract Res 2006 Dec;36(4):318-24.

10. Blazeby J. Do dressings prevent infection of closed primary wounds after surgery? BMJ (Online) 2016 May;24:353.

11. Vowden K, Vowden P. Wound dressings: principles and practice. Surgery (Oxford) 2017 Sep 1;35(9):489-94.

12. Bernatchez SF. Care of peripheral venous catheter sites: advantages of transparent film dressings over tape and gauze. J Assoc Vascular Access 2014 Dec 1;19(4):256-61.

13. Alavi A, Sibbald RG, Ladizinski B, Saraiya A, Lee KC, Skotnicki-Grant S et al. Wound-related allergic/irritant contact dermatitis. Adv Skin Wound Care 2016 Jun 1;29(6):278-86.

14. Morris C, Emsley P, Marland E, Meuleneire F, White R. Use of wound dressings with soft silicone adhesive technology. Nurs Child Young People 2009 Apr 27;21(3).

15. McNichol L, Lund C, Rosen T, Gray M. Medical adhesives and patient safety: state of the science consensus statements for the assessment, prevention, and treatment of adhesive-related skin injuries. J Dermatol Nurses' Assoc 2013 Nov 1;5(6):323-38.

16. LeBlanc K, Campbell K, Beeckman D, Dunk A, Harley C, Hevia H, et al. Best practice recommendations for the prevention and management of skin tears in aged skin. Wounds International [Internet] 2018. Available from: https://www.woundsinternational.com/resources/details/istapbest-practice-recommendations-prevention-and-management-skintears-aged-skin

17. McNichol L, Bianchi J. MARSI made easy. Wounds UK [Internet] 2016 Nov;12(4). Available from: https://www.wounds-uk.com/resources/ details/medical-adhesive-related-skin-injuries-marsi-made-easy 